\title{
Risk Stratification of Thyroid Nodules Using the Thyroid Imaging Reporting and Data System (TIRADS): The Omission of Thyroid Scintigraphy Increases the Rate of Falsely Suspected Lesions
}

\author{
Simone Schenke ${ }^{1}$, Philipp Seifert ${ }^{2}$, Michael Zimny ${ }^{1}$, Thomas Winkens ${ }^{2}$, Ina Binse ${ }^{3}$, and Rainer Görges ${ }^{3,4}$ \\ ${ }^{1}$ Institute of Nuclear Medicine Hanau, Giessen, Germany; ${ }^{2}$ Clinic of Nuclear Medicine, Jena University Hospital, Jena, Germany; \\ ${ }^{3}$ Clinic of Nuclear Medicine, Essen University Hospital, Essen, Germany; and ${ }^{4}$ Group Practice for Nuclear Medicine Duisburg/ \\ Moers, Duisburg, Germany
}

\begin{abstract}
Thyroid nodules are a common finding, especially in iodine-deficient regions. Ultrasonographic scoring systems such as the Thyroid Imaging Reporting and Data System (TIRADS) are helpful in differentiating between benign and malignant thyroid nodules by offering a risk stratification model. Depending on the constellation or number of suspicious ultrasound features, a fine-needle biopsy is recommended. However, none of the previous TIRADS publications considered the functional status of the nodules. Hyperfunctioning thyroid nodules (HTNs) were presumed to exclude malignancy with a very high negative predictive value. Particularly in regions where the iodine supply is low, most HTNs are seen in patients with normal thyroid-stimulating hormone levels. Therefore, thyroid scintigraphy is essential for the detection of HTNs. We investigated whether TIRADS identifies HTNs as nonsuspicious. Methods: We evaluated 615 HTNs $(23.2 \pm 10.0 \mathrm{~mm}$ in maximum diameter in 582 patients ([442 women, $57.7 \pm 13.2$ y old, and 140 men, $60.1 \pm 12.7$ y old) detected by ${ }^{99 m}$ Tc-pertechnetate or ${ }^{123}$ s scintigraphy. Before evaluating the scintigraphic appearance, all nodules were analyzed prospectively with sonography, using the TIRADS model referenced in Kwak et al., wherein fine-needle biopsy is recommended for TIRADS 4A or higher. We also investigated 2 subgroups, 42 nodules with available histology and 117 patients with subclinical or overt hyperthyroidism. Results: Whereas $15.9 \%$ of the nodules were classified as TIRADS 3 or lower and less than $0.1 \%$ as TIRADS 5 , most of the nodules were classified as TIRADS 4A (29.3\%), 4B (29.3\%), or $4 \mathrm{C}(24.9 \%)$. Altogether, more than $80 \%$ of the autonomous thyroid nodules were classified as TIRADS 4A or higher, a grade that would result in a recommendation of fine-needle biopsy. Focusing on those 117 HTNs that were already associated with hyperthyroid laboratory values, the rates were similar: $81.2 \%$ were categorized as TIRADS 4A or higher (4A, 33.3\%; 4B, 29.9\%; $4 C, 17.1 \% ; 5,0.9 \%)$. In the subgroup of patients who underwent thyroid surgery, all nodules were benign, confirming the known negative predictive value of HTNs with regard to malignancy exclusion. Conclusion: Integration of thyroid scintigraphy into the TIRADS model is essential to prevent unnecessary fine-needle biopsy and thyroid surgery.
\end{abstract}

Received Mar. 27, 2018; revision accepted Jul. 20, 2018.

For correspondence or reprints contact: Simone Schenke, Institute of Nuclear Medicine Hanau, Paul-Zipp-Straße 171, 35398 Gießen, Germany.

E-mail: simoneschenke@web.de

Published online Aug. 10, 2018.

COPYRIGHT (C 2019 by the Society of Nuclear Medicine and Molecular Imaging.
Key Words: TIRADS; thyroid nodules; hyperfunctioning thyroid nodules; thyroid scintigraphy

J Nucl Med 2019; 60:342-347

DOI: 10.2967/jnumed.118.211912

$\mathbf{T}$ he prevalence of thyroid nodules is high, especially in iodine-deficient countries. Germany is a country with a long history of iodine deficiency; even today, the iodine supply is considered suboptimal (1). In general, the prevalence of thyroid nodules in adults without previously diagnosed thyroid disorders was estimated at up to $59 \%$, with a higher prevalence in the older population $(2,3)$. Furthermore, multinodularity is a common finding in patients with thyroid nodules $(3,4)$. However, only a small number of these nodules are of clinical relevance. Consequently, the official journal of the German Medical Association published a statement against sonographic screening for thyroid nodules in elderly asymptomatic patients (5). A comparable recommendation against screening in asymptomatic adults was published in 2017 by the U.S. Preventive Services Task Force (6). But, on the other hand, thyroid nodules are a common incidental finding in imaging studies of the neck, including duplex sonography of the arteries, MRI or CT, as well as ultrasonography in the context of otolaryngologic examinations. Thus, it is necessary to decide which nodules pose a potential risk and require further work-up to detect either those suspected of being malignant (fine-needle biopsy, thyroid surgery) or those that are functionally relevant autonomous nodules. Official guidelines emphasize the importance of high-resolution ultrasound for an accurate assessment of sonomorphologic nodule features. B-mode ultrasound can be combined with colorduplex to assess the vascularity and, more recently, with sonoelastography to assess the elasticity or stiffness of thyroid nodules $(7,8)$.

In the past, several ultrasound characteristics indicative of malignant or benign nodules were identified (9-11). However, there are still no universally applied standardized guidelines for the use and weighing of these characteristics. Thus, it is of interest to achieve a more standardized risk assessment of thyroid nodules by using different combinations of these single criteria and to establish a Thyroid Imaging Reporting and Data System (TIRADS) 
comparable to the Breast Imaging and Data Reporting System first introduced by the American College of Radiology in 1997 (12). Horvath et al. published the first TIRADS in 2009, consisting of an atlas of typical thyroid nodules (13). A patient's nodule is classified by comparing it with this set of standard nodules. This process is difficult to integrate into clinical routine and does not cover all types of thyroid nodules $(14,15)$. More recently, several further TIRADS versions were introduced, including the Korean-TIRADS (16-18), based on the modified publication by Kwak et al. in 2011 and 2013 (19,20). Basically, in the Kwak-TIRADS a sum score for each thyroid nodule according to 5 defined sonographic nodule features is calculated to determine the need for fine-needle biopsy. This simple scoring system is more applicable to the classification of thyroid nodules in a clinical setting $(14,15,18)$. The American College of Radiology presented a more sophisticated TIRADS in 2015 $(21,22)$, and the EU-TIRADS by the European Thyroid Association followed in 2017 (23). Two recent publications compared the various TIRADS versions and described an overall agreement $(24,25)$.

However, at present, none of the TIRADS versions address the functional status of thyroid nodules, despite the fact that the finding of a scintigraphically hot, or hyperfunctioning, thyroid nodule (HTN) has a high negative predictive value for malignancy. Critical analyses of reports of thyroid carcinoma occurring as HTNs on scintigraphy showed that this finding is a rare phenomenon and that further investigation to exclude malignancy is not required (26-28). Walfish et al. evaluated the diagnostic and treatment consequences of using a routine fine-needle biopsy of HTNs. Among other findings, nuclear pleomorphism found in some nodules raised suspicion of malignancy. They concluded that a total reliance on fine-needle biopsy without performing scintigraphy would result in surgery for benign nodules suspected of being malignant and may increase the surgical morbidity (29). Moreover, a systematic exclusion of malignancy in HTNs with fine-needle biopsy is presumed to not be cost-effective (30). Furthermore, HTNs cannot reliably be identified or excluded by the thyroid-stimulating hormone (TSH) level alone (31). In Germany, for example, most HTNs are seen in patients with normal TSH levels $(32,33)$.

The aim of this prospective study was to evaluate KwakTIRADS in HTNs. We addressed the question of whether standalone TIRADS is suitable to correctly classify these nodules as benign or whether scintigraphy should be added to the diagnostic work-up of thyroid nodules.

\section{MATERIALS AND METHODS}

\section{Patients and Study Design}

This prospective study was approved by the appropriate ethical committee and Institutional Review Board (16-7022B0), and the requirement to obtain informed consent was waived. From 2012 to 2017, 1,719 thyroid nodules (entire cohort) were investigated at the 3 nuclear medicine institutions in Germany (an outpatient practice in the middle of Germany [site 1], an outpatient practice in the west [site 2], and a university hospital in the east [site 3]). Thyroid ultrasound was performed on all patients, and the dominating nodules were scored and categorized according to the Kwak-TIRADS (19). For nodules exceeding the cutoff of $10 \mathrm{~mm}$, thyroid scintigraphy was routinely performed. For the current study, we evaluated only a subgroup of HTNs $(n=615)$. The perifocal thyroid tissue was scintigraphically more or less suppressed. Scintigraphically hypofunctioning or indifferent thyroid nodules were excluded. The routinely recorded data from all patients were sex; age; thyroid-related medications; thyroid function parameters such as TSH, free thyroxine, free triiodothyronine, and thyroid peroxidase-autoantibodies; and, in the case of surgery, the histopathologic results. The primary indication for surgery was the patients' decision against radioiodine therapy.

\section{Ultrasound}

Ultrasound of the thyroid gland was performed using a Hitachi EUB $5000 \mathrm{G}$ or a Hitachi Avius Hi Vision equipped with a linear probe with a frequency of $5-10 \mathrm{MHz}$ (site 1), a Mindray DC-6 or an Esaote MyLab 40 equipped with small-part probes with frequencies of 10 and $12 \mathrm{MHz}$ (site 2), and a GE Healthcare Logiq E9 equipped with a linear probe with a frequency of $6-15 \mathrm{MHz}$ (site 3). The standard ultrasound protocol in all cases included volumetry of the thyroid gland $\left(\mathrm{cm}^{3}\right)$, the size of the detected nodules (width, depth, and length, in $\mathrm{mm}$ ), and the position of the nodules within the lobes, for matching with the scintigraphic image. The Kwak-TIRADS classification of the nodules was done without knowledge of the scintigraphic pattern of the nodules. The following ultrasound features were assessed in all patients for each detected nodule: composition (solid composition with or without cystic components), echogenicity of the solid component (hyperechoic/isoechoic/hypoechoic/marked hypoechoic), calcifications (macrocalcification/microcalcification), margin (smooth/lobulated/illdefined or blurred), and shape (taller-than-wide/wider-than-tall). Accounting for the number of suspicious ultrasound features (solid composition without cystic components, hypoechogenicity/marked hypoechogenicity, microcalcifications, lobulated or ill-defined margin, taller-than-wide shape) present in the nodule, we classified the nodules according to the Kwak-TIRADS as TIRADS 3 (no suspicious features), TIRADS 4A ( 1 suspicious feature), TIRADS 4B ( 2 suspicious features), TIRADS 4C ( 3 or 4 suspicious features), or TIRADS 5 ( 5 suspicious features) (19). A spongiform or an anechoic (pure cystic) nodule was classified as TIRADS 2 (13).

\section{Scintigraphy and Serum Parameters}

For nodules with a maximum diameter of $10 \mathrm{~mm}$, standardized scintigraphy was performed according to guidelines $(34,35)$. Site 1 used a Mediso Nucline Spirit, a low-energy high-resolution collima-

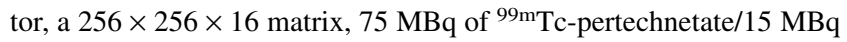
of ${ }^{123}$ I-iodide, and an acquisition time of 5 or $10 \mathrm{~min}$. Site 2 used a Philips CX250S, a low-energy high-resolution collimator, a $128 \times 128$ matrix, $75 \mathrm{MBq}$ of ${ }^{99 \mathrm{~m}} \mathrm{Tc}$-pertechnetate, and an acquisition time of 5$10 \mathrm{~min}$. Site 3 used a Mediso Nucline TH, a low-energy high-resolution collimator, a $128 \times 128$ matrix, $70 \mathrm{MBq}$ of ${ }^{99 \mathrm{~m} T c-p e r t e c h n e t a t e,}$ and an acquisition time of $4 \mathrm{~min}$. The distribution of the tracer within the thyroid gland was correlated with the ultrasound findings. Nodules with a visible focal increase in uptake compared with the adjacent thyroid tissue were defined as hyperfunctioning. Uptake measurements were not analyzed.

TSH was determined with various commercially available immunometric assays. depending on the laboratory. For TSH, the lower level of the reference range of the respective laboratories ranged from 0.20 to $0.35 \mathrm{mU} / \mathrm{L}$. In a separate analysis of patients without thyroidrelated medication, TSH values below the respective laboratory reference range were classified as hyperthyroidism (as overt hyperthyroidism if there was a simultaneous increase in free thyroxine or free triiodothyronine; otherwise, as subclinical hyperthyroidism).

\section{Statistics}

All statistical tests were performed using WinSTAT for Microsoft Excel, version 2005.1, and IBM SPSS Statistics, version 24. The results were expressed as mean, $\mathrm{SD}$, median, and range. The variables were tested using the $t$ test, $\chi^{2}$ test, or ANOVA as appropriate. All results were considered to be significant at a $P$ value of less than 0.05 (adjusted $P$ value, Holm-Bonferroni adjustment). 
TABLE 1

Patient Characteristics and Ultrasonographic Appearance of Thyroid Nodules at All Investigation Sites

\begin{tabular}{|c|c|c|c|c|c|c|}
\hline Characteristic & Total & Site 1 & Site 2 & Site 3 & Test & $P$ \\
\hline Patients & $582(100)$ & $145(24.9)$ & $300(51.5)$ & $137(23.6)$ & $\chi^{2}$ & 0.0002 \\
\hline Female & $442(75.9)$ & $115(79.3)$ & $246(82.0)$ & $81(59.1)$ & & \\
\hline Male & $140(24.1)$ & $30(20.7)$ & $54(18.0)$ & $56(40.9)$ & & \\
\hline Mean age (y) & $58.3 \pm 13.1$ & $55.4 \pm 13.4$ & $57.5 \pm 13.4$ & $62.9 \pm 10.8$ & ANOVA & 0.0002 \\
\hline Female & $57.7 \pm 13.2$ & $55.8 \pm 13.7$ & $57.1 \pm 13.3$ & $62.3 \pm 11.0$ & & 0.0231 \\
\hline Male & $60.1 \pm 12.7$ & $54.1 \pm 12.4$ & $59.6 \pm 13.7$ & $63.7 \pm 10.6$ & & 0.0426 \\
\hline Nodules & $615(100)$ & $145(23.6)$ & $317(51.5)$ & $153(24.9)$ & - & - \\
\hline Median largest diameter (mm) & $23.2 \pm 10.0$ & $22.4 \pm 8.7$ & $21.8 \pm 9.2$ & $27.3 \pm 11.6$ & ANOVA & 0.0002 \\
\hline Patients with hyperthyroidism & $117(20.1)$ & $21(14.5)$ & $56(18.7)$ & $40(29.2)$ & $\chi^{2}$ & 1.0000 \\
\hline Resected nodules & $42(6.8)$ & $30(20.7)$ & $10(3.2)$ & $2(1.3)$ & $\chi^{2}$ & $<0.0001$ \\
\hline \multicolumn{7}{|l|}{ Ultrasound features } \\
\hline Solid composition & $348(56.6)$ & $77(53.1)$ & $190(59.9)$ & $81(52.9)$ & $\chi^{2}$ & 1.0000 \\
\hline Hypoechogenicity & $311(50.6)$ & 67 (46.2) & $169(53.3)$ & $75(49.0)$ & $x^{2}$ & 1.0000 \\
\hline Marked hypoechogenicity & $34(5.5)$ & $9(6.2)$ & $15(4.7)$ & $10(6.5)$ & $\chi^{2}$ & 1.0000 \\
\hline Calcification & 263 (42.8) & $43(29.7)$ & $151(47.6)$ & $69(45.1)$ & $x^{2}$ & 0.0168 \\
\hline Microcalcification & $209(34.0)$ & $31(21.4)$ & $130(41.0)$ & $48(31.4)$ & $\chi^{2}$ & 0.0021 \\
\hline Irregular margins & $117(19.0)$ & $33(22.8)$ & $58(18.3)$ & $26(17.0)$ & $\chi^{2}$ & 1.0000 \\
\hline Lobulated margins & $62(10.1)$ & $17(11.7)$ & $31(9.8)$ & $14(9.2)$ & $\chi^{2}$ & 1.0000 \\
\hline III-defined margins & $55(8.9)$ & $16(11.0)$ & $27(8.5)$ & $12(7.8)$ & $\chi^{2}$ & 1.0000 \\
\hline Taller than wide shape & $66(10.7)$ & $21(14.5)$ & $35(11.0)$ & $10(6.5)$ & $\chi^{2}$ & 1.0000 \\
\hline
\end{tabular}

Qualitative data are expressed as numbers followed by percentages in parentheses; continuous data are expressed as mean or median \pm SD.

\section{RESULTS}

In total, 615 HTNs in 582 patients (442 women, 140 men) with an average age of $58.3 \pm 13.1 \mathrm{y}$ (range, 19-91 y) were included in this study (Table 1). The mean size of the nodules was $23.2 \pm$ $10.0 \mathrm{~mm}$.

The database comprised 145 patients with 145 thyroid nodules at site 1, 300 patients with 317 nodules at site 2 , and 137 patients with 153 nodules at site 3 . Thyroid-related medication was taken by $198(34.0 \%)$ patients (iodide, $n=14$ [supplemental dosage, not discontinued before scan]; thyroid hormones, $n=149$; a combination of iodide [supplemental dosage] and thyroid hormones, $n=$ 27; antithyroid drugs, $n=8$ ).

In comparison with the other sites, site 1 had a lower percentage of nodules with calcifications $(P=0.0168)$ and patients underwent surgery more often (a total of 30 resected nodules at site 1 , $P<0.0001)$. At site 3 , the proportion of men was higher $(P=$ $0.0002)$ and the patients were older at the time of investigation $(P=0.0002)$. Furthermore, the nodules had larger diameters at site $3(P=0.0002)$ (Table 1$)$.

The Kwak-TIRADS classification revealed 98 (5.9\%), 180 (29.3\%), $180(29.3 \%), 153(24.9 \%)$, and $4(0.01 \%)$ nodules in categories $\leq 3,4 \mathrm{~A}, 4 \mathrm{~B}, 4 \mathrm{C}$, and 5 , respectively (Fig. 1). Category 4C included 118 (19.2\%) nodules with 3 of 5 ultrasound features and $35(5.7 \%)$ nodules with 4 of 5 ultrasound features. The number of nodules classified as 4A or higher was 517 (83.5\%). Solid composition, hypoechogenicity, microcalcification, irregular margins, and a taller-than-wide shape was found in 348 (56.6\%), 311 (50.6\%), 209 (34.0\%), $117(19.0 \%)$, and $66(10.7 \%)$ nodules, respectively. None of the $42(6.8 \%)$ thyroid nodules in $42(7.2 \%)$ patients who underwent surgery were malignant.

The median TSH level at the time of scintigraphy for all patients was $0.56 \mathrm{mU} / \mathrm{L}$ (TSH range, $0.01-6.4 \mathrm{mU} / \mathrm{L}$ ). For patients without thyroid-related medication $(n=430)$, the median TSH level was 0.62 (range, 0.01-5.72 mU/L). Subclinical or overt hyperthyroidism was seen in $117(20.1 \%)$ patients (Kwak-TIRADS category $\leq 3,22$ patients [18.8\%]; 4A, 39 patients [33.3\%]; 4B, 35

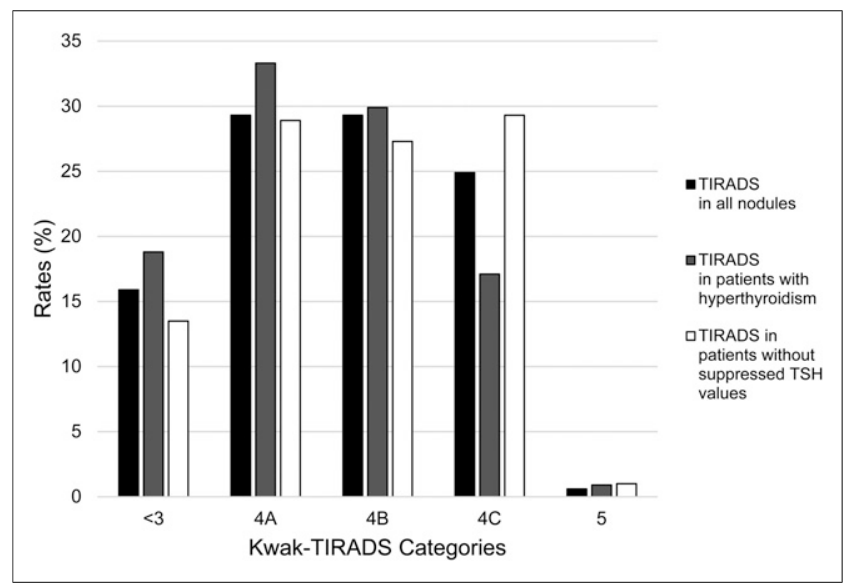

FIGURE 1. Distribution of thyroid nodules (all patients, patients with hyperthyroidism [no medication], and patient without suppressed TSH [no medication]) for TIRADS categories. 
patients [29.9\%]; 4C, 20 patients [17.1\%]; and 5, 1 patient [0.9\%]). A similar distribution $(P=0.433026$, Fisher exact test) of KwakTIRADS categories was found for the 311 euthyroid patients who did not take any thyroid medications: Kwak-TIRADS category $\leq 3,42$ patients (13.5\%); 4A, 90 patients (28.9\%); 4B, 85 patients $(27.3 \%)$; 4C, 91 patients (29.3\%); and 5, 3 patients (1\%) (Fig. 1).

Representative examples of the different TIRADS categories are shown in Figure 2.

\section{DISCUSSION}

To our knowledge, this was the first prospective study to evaluate TIRADS in HTNs. Despite the fact that a hot thyroid nodule has a negligible risk of malignancy, the scintigraphic appearance of thyroid nodules has not yet been addressed in studies on TIRADS classifications. In Germany, autonomous nodules are still a common finding, with a prevalence of approximately $21 \%$ of unselected thyroid nodules when evaluated with
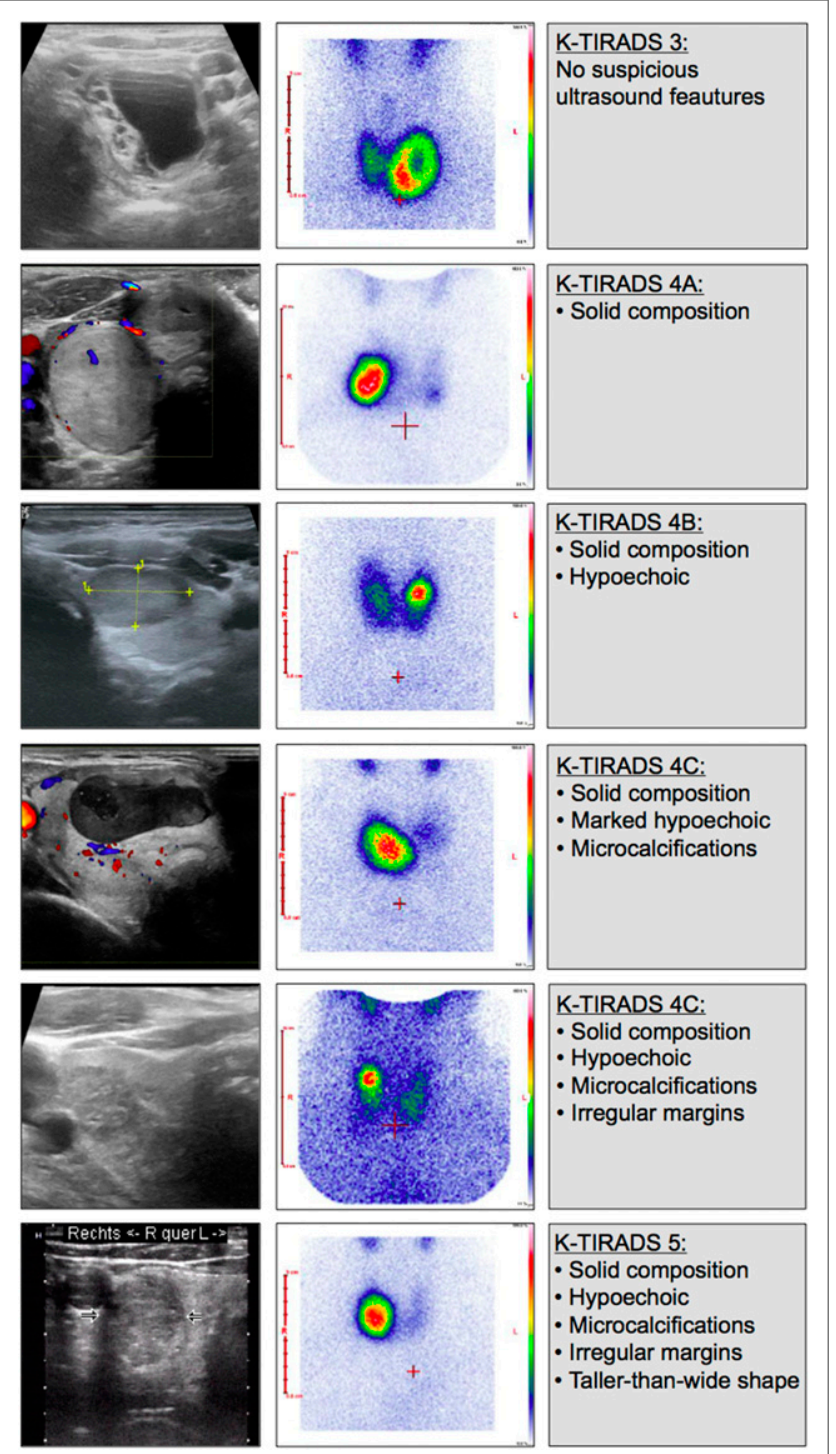

FIGURE 2. Examples for sonographic appearance and TIRADS category of HTNs. scintigraphy $(33,36)$. Analyzing a representative sample of consecutive patients presenting for the first time for thyroid diagnostics, we found similar rates of HTNs: 20\% (site 1, 217 evaluated patients) or $21 \%$ (site 2, 81 evaluated patients). Therefore, the guidelines of the German Society of Nuclear Medicine (37) and of the German Association of Endocrine Surgeons recommend supplementary scintigraphy for nodules larger than $1 \mathrm{~cm}$ independently of the TSH level (38), whereas the current guidelines of the American Thyroid Association recommend against the routine use of scintigraphy in the diagnostic workup of thyroid nodules $(7,8,39)$.

Our results show that a large number of HTNs were classified as TIRADS $4 \mathrm{~A}$ or higher $(83.5 \%)$ and that close to a quarter of the nodules were classified as TIRADS 4C. According to the study by Kwak et al., the former corresponds to a risk of malignancy of $3.3 \%$ and the latter to a risk of $44 \%-72 \%$ (19), requiring cytologic or even histologic exclusion of malignancy. Most nodules in this study (with a Kwak-TIRADS score of 4A or higher) had a solid composition, hypoechogenicity occurred in about half, microcalcifications in about a third, and irregular margins in about $20 \%$ of the nodules. However, taking into account the scintigraphic finding of a hot nodule, these nodules are most probably benign, as underlined by the fact that no malignancy was found in the subgroup of patients who underwent thyroid surgery in this study.

Recently, Ruhlmann et al. (40) evaluated ultrasound elastography in 135 autonomous nodules and showed that $70 \%$ of these nodules were classified as hard. Thus, even the inclusion of elastography in ultrasonographic assessment of nodules cannot replace the diagnostic information of scintigraphy (40). This postulate is supported by another recent study (41).

Current guidelines from various thyroid associations give only a minor role to scintigraphy in evaluating thyroid nodules and recommend scintigraphy only in patients with low TSH (8). However, in regions with iodine deficiency, the indication for scintigraphy should be independent of the TSH level as stated by the guidelines for clinical practice for the diagnosis and management of thyroid nodules of the American Association of Clinical Endocrinologists, the Associazione Medici Endocrinologi, and the European Thyroid Association (39). This recommendation takes into account that the prevalence of hyperthyroidism and, thus, a suppressed TSH concentration, in patients with HTNs depends on the iodine supply, which is still suboptimal in several European countries, including Germany (1). Two studies from Germany reported that most HTNs are found in patients with a TSH level within the reference range $(32,33)$. This observation was supported by studies from other European and non-European countries with a better iodine supply, as summarized in a review by Treglia et al. (31). On average, about half the patients with HTNs had a normal TSH. Thus, TSH alone cannot reliably detect all HTNs.

As a rule, the finding of a hot nodule on scintigraphy represents an autonomous nodule. However, the phenomenon of a trappingonly nodule may occur in 5\% of HTNs as shown in a prospective study of 140 patients with normal thyroid function. In their study, Reschini et al. described that 5 of 7 trapping-only nodules were benign whereas 2 were malignant (42). A trapping-only nodule should be considered if a large nodule is seen in a patient with a relatively high TSH concentration. To investigate this aspect, we analyzed a subgroup of 117 HTNs with endogenously suppressed $\mathrm{TSH}$, indicating subclinical or overt hyperthyroidism and a correspondingly low uptake by the perinodular thyroid tissue on scintigraphy, most certainly excluding trapping-only nodules. In this subgroup of autonomous nodules, the percentage of nodules classified 
as Kwak-TIRADS 4A or higher was comparable to the results for all patients and to the results for the patients who were euthyroid without taking thyroid medications.

For the classification of thyroid nodules with ultrasound, we used the TIRADS introduced by Kwak et al. (19). This system assigns a score based on the number of 5 different suspicious B-mode ultrasound features that are present in a nodule. Such a system allows for a fast learning process and easy integration of TIRADS into clinical routine (14). As a limitation, we did not apply other TIRADS versions. However, recent studies addressed the comparability of Kwak-TIRADS, American College of Radiology-TIRADS, and EUTIRADS and reported good agreement $(24,25)$. Thus, it is unlikely that other classification systems based on ultrasound alone would have revealed different results for autonomous thyroid nodules. As another limitation, we did not calculate the interobserver variability of the Kwak-TIRADS. Nevertheless, in this study, the reproducibility of Kwak-TIRADS was acceptable, with classification showing a high level of agreement as tested on control files that were evaluated at each site (data not shown). In addition, the distribution of ultrasound characteristics at the 3 sites was similar, with significant differences found only for the frequency of microcalcifications. In clinical routine, it is rather difficult to distinguish real microcalcification from so-called hyperechogenic foci, which are usually a benign finding. Especially in nodules that are predominantly solid, the comet tail sign of the hyperechogenic foci may be difficult to identify. Therefore, the lower frequency of microcalcifications most likely can be attributed to interobserver variability.

\section{CONCLUSION}

On the basis of the results of the present study, we recommend integration of scintigraphy into the TIRADS model. Detection of HTNs can reduce the number of unnecessary diagnostic procedures such as fine-needle biopsy or-in the extreme-diagnostic surgery. Including the functional classification of sonographically suspicious thyroid nodules improves the positive predictive value of a high TIRADS class. Nonetheless, scintigraphy is still the method of choice to assess the functional relevance of autonomous nodules and, thus, the risk to develop hyperthyroidism after exposure to high amounts of iodine. Our conclusion regarding the importance of scintigraphy agrees with publications by other authors (43-46).

\section{DISCLOSURE}

No potential conflict of interest relevant to this article was reported.

\section{ACKNOWLEDGMENTS}

We thank Dr. Kelsey L. Pomykala of the Department of Radiology at UCLA Medical Center for language editing, and we thank PD Dr. med. Martin Freesmeyer of the Clinic of Nuclear Medicine at Jena University Hospital for providing access to patient data and images.

\section{REFERENCES}

1. Johner SA, Thamm M, Schmitz R, Remer T. Examination of iodine status in the German population: an example for methodological pitfalls of the current approach of iodine status assessment. Eur J Nutr. 2016;55:1275-1282.

2. Meisinger $\mathrm{C}$, Ittermann $\mathrm{T}$, Wallaschofski $\mathrm{H}$, et al. Geographic variations in the frequency of thyroid disorders and thyroid peroxidase antibodies in persons without former thyroid disease within Germany. Eur J Endocrinol. 2012;167:363371.

3. Carlé A, Krejbjerg A, Laurberg P. Epidemiology of nodular goiter: influence of iodine intake. Best Pract Res Clin Endocrinol Metab. 2014;28:465-479.
4. Dean DS, Gharib H. Epidemiology of thyroid nodules. Best Pract Res Clin Endocrinol Metab. 2008;22:901-911.

5. Feldkamp J, Schott M, Quinkler M, Blüher M, Diederich S, Reincke M. Deciding wisely... in endocrinology [in German]. Dtsch Arztebl. 2016;113:A821A824.

6. US Preventive Services Task Force, Bibbins-Domingo K, Grossman DC, et al. Screening for Thyroid Cancer: US Preventive Services Task Force Recommendation Statement. JAMA. 2017;317:1882-1887.

7. Perros P, Boelaert K, Colley S, et al. Guidelines for the management of thyroid cancer. Clin Endocrinol (Oxf). 2014;81(suppl 1):1-122.

8. Haugen BR, Alexander EK, Bible KC, et al. American Thyroid Association management guidelines for adult patients with thyroid nodules and differentiated thyroid cancer: the American Thyroid Association Guidelines task force on thyroid nodules and differentiated thyroid cancer. Thyroid. 2016;26: $1-133$.

9. Brito JP, Gionfriddo MR, Al Nofal A, et al. The accuracy of thyroid nodule ultrasound to predict thyroid cancer: systematic review and meta-analysis. $J$ Clin Endocrinol Metab. 2014;99:1253-1263.

10. Campanella P, Ianni F, Rota CA, Corsello SM, Pontecorvi A. Quantification of cancer risk of each clinical and ultrasonographic suspicious feature of thyroid nodules: a systematic review and meta-analysis. Eur J Endocrinol. 2014;170: R203-R211.

11. Remonti LR, Kramer CK, Leitão CB, Pinto LC, Gross JL. Thyroid ultrasound features and risk of carcinoma: a systematic review and meta-analysis of observational studies. Thyroid. 2015;25:538-550.

12. D'Orsi CJ, Sickles EA, Mendelson EB, et al. ACR BI-RADS Atlas, Breast Imaging Reporting and Data System. Reston, VA, American College of Radiology; 2013.

13. Horvath E, Majlis S, Rossi R, et al. An ultrasonogram reporting system for thyroid nodules stratifying cancer risk for clinical management. J Clin Endocrinol Metab. 2009;94:1748-1751.

14. Schenke S, Rink T, Zimny M. TIRADS for sonographic assessment of hypofunctioning and indifferent thyroid nodules. Nuklearmedizin. 2015;54:144-150.

15. Wang Y, Lei KR, He YP, et al. Malignancy risk stratification of thyroid nodules: comparisons of four ultrasound thyroid imaging reporting and data systems in surgically resected nodules. Sci Rep. 2017;7:11560.

16. Ha EJ, Moon WJ, Na DG, et al. A multicenter prospective validation study for the Korean thyroid imaging reporting and data system in patients with thyroid nodules. Korean J Radiol. 2016;17:811-821.

17. Park JW, Kim DW, Kim D, Baek JW, Lee YJ, Baek HJ. Korean thyroid imaging reporting and data system features of follicular thyroid adenoma and carcinoma: a single-center study. Ultrasonography. 2017;36:349-354.

18. Migda B, Migda M, Migda MS, Slapa RZ. Use of the Kwak thyroid image reporting and data system (K-TIRADS) in differential diagnosis of thyroid nodules: systematic review and meta-analysis. Eur Radiol. 2018;28:23802388.

19. Kwak JY, Han KH, Yoon JH, et al. Thyroid imaging reporting and data system for US features of nodules: a step in establishing better stratification of cancer risk. Radiology. 2011;260:892-899.

20. Kwak JY, Jung I, Baek JH, et al. Image reporting and characterization system for ultrasound features of thyroid nodules: multicentric Korean retrospective study. Korean J Radiol. 2013;14:110-117.

21. Grant EG, Tessler FN, Hoang JK, et al. Thyroid ultrasound reporting lexicon: white paper of the ACR thyroid imaging, reporting and data system (TIRADS) committee. J Am Coll Radiol. 2015;12:1272-1279.

22. Tessler FN, Middleton WD, Grant EG, et al. ACR thyroid imaging, reporting and data system (TI-RADS): white paper of the ACR TI-RADS committee. J Am Coll Radiol. 2017;14:587-595.

23. Russ G, Bonnema SJ, Erdogan MF, Durante C, Ngu R, Leenhardt L. European Thyroid Association guidelines for ultrasound malignancy risk stratification of thyroid nodules in adults: the EU-TIRADS. Eur Thyroid J. 2017;6:225-237.

24. Ha SM, Ahn HS, Baek JH, et al. Validation of three scoring risk-stratification models for thyroid nodules. Thyroid. 2017;27:1550-1557.

25. Grani G, Lamartina L, Cantisani V, Maranghi M, Lucia P, Durante C. Interobserver agreement of various thyroid imaging reporting and data systems. Endocr Connect. 2018;7:1-7.

26. Schröder S, Marthaler B. Autonomy and malignancy of thyroid gland tumors: a critical analysis of the literature on the existence of hyperfunctioning follicular and papillary thyroid gland carcinomas. Pathologe. 1996;17:349-357.

27. Lupi A, Orsolon P, Cerisara D, Deantoni Migliorati G, Vianello Dri A. "Hot" carcinoma of the thyroid: case reports and comments on the literature. Minerva Endocrinol. 2002;27:53-57.

28. Bomeli SR, LeBeau SO, Ferris RL. Evaluation of a thyroid nodule. Otolaryngol Clin North Am. 2010;43:229-238. 
29. Walfish PG, Strawbridge HT, Rosen IB. Management implications for routine needle biopsy of hyperfunctioning thyroid nodules. Surgery. 1985;98:11791188 .

30. Lima MJ, Soares V, Koch P, Silva A, Taveira-Gomes A. Autonomously hyperfunctioning cystic nodule harbouring thyroid carcinoma: case report and literature review. Int J Surg Case Rep. 2018;42:287-289.

31. Treglia G, Trimboli P, Verburg FA, Luster M, Giovanella L. Prevalence of normal TSH value among patients with autonomously functioning thyroid nodule. Eur J Clin Invest. 2015;45:739-744.

32. Görges R, Kandror T, Kuschnerus S, et al. Scintigraphically "hot" thyroid nodules mainly go hand in hand with a normal TSH. Nuklearmedizin. 2011;50:179188.

33. Graf D, Helmich-Kapp B, Graf S, Veit F, Lehmann N, Mann K. Functional activity of autonomous adenoma in Germany. Dtsch Med Wochenschr. 2012;137: 2089-2092.

34. Radionuclide thyroid scans: report 2003. British Nuclear Medicine Society website. https://www.bnms.org.uk/images/stories/Procedures_and_Guidelines/radionuclide_thyroid_scans_2016.pdf. Published April 2001. Revised April 2005. Accessed October 25, 2018

35. ACR-SPR practice parameter for the performance of scintigraphy and uptake measurements for benign and malignant thyroid disease. American College of Radiology website. https://www.acr.org/-/media/ACR/Files/Practice-Parameters/ Thy-Scint.pdf. Published 1995. Last revised 2014. Accessed October 25, 2018

36. Luster M, Führer D, Engelhardt C, et al. Prevalence of scintigraphically hot, cold and indifferent thyroid nodules in patients with a known solitary lesion [in German]. Nuklearmedizin. 2005;44:A6-A7.

37. Dietlein M, Eschner W, Lassmann M, Verburg F, Luster M. Procedure guideline for thyroid scintigraphy (version 4) [in German]. German Society for Nuclear Medicine website http://www.nuklearmedizin.de/leistungen/leitlinien/docs/0310111_S1_Schilddruesenszintigraphie_2014-10.pdf. Published October 2014. Accessed October 25, 2018
38. Dralle H, Musholt TJ, Schabram J, et al.; German Societies of General and Visceral Surgery, Endocrinology, Nuclear Medicine, Pathology, Radiooncology, and Oncological Hematology and the German Thyroid Cancer Patient Support Organization Ohne Schilddrüse leben e.V. German Association of Endocrine Surgeons practice guideline for the surgical management of malignant thyroid tumors. Langenbecks Arch Surg. 2013;398:347-375.

39. Gharib H, Papini E, Garber JR, et al.; AACE/ACE/AME Task Force on Thyroid Nodules. American Association of Clinical Endocrinologists, American College of Endocrinology, and Associazione Medici Endocrinologi medical guidelines for clinical practice for the diagnosis and management of thyroid nodules: 2016 Update. Endocr Pract. 2016;22:622-639.

40. Ruhlmann M, Stebner V, Görges R, et al. Diagnosis of hyperfunctional thyroid nodules: impact of US-elastography. Nuklearmedizin. 2014;53:173-177.

41. Trimboli P, Paone G, Zatelli MC, Ceriani L, Giovanella L. Real-time elastography in autonomously functioning thyroid nodules: relationship with TSH levels, scintigraphy, and ultrasound patterns. Endocrine. 2017;58:488-494.

42. Reschini E, Ferrari C, Castellani M, et al. The trapping-only nodules of the thyroid gland: prevalence study. Thyroid. 2006;16:757-762.

43. Ianni F, Perotti G, Prete A, et al. Thyroid scintigraphy: an old tool is still the gold standard for an effective diagnosis of autonomously functioning thyroid nodules. J Endocrinol Invest. 2013;36:233-236.

44. Giovanella L, Ceriani L, Treglia G. Role of isotope scan, including positron emission tomography/computed tomography, in nodular goitre. Best Pract Res Clin Endocrinol Metab. 2014;28:507-518.

45. Garberoglio S, Testori O. Role of nuclear medicine in the diagnosis of benign thyroid diseases. Front Horm Res. 2016;45:24-36.

46. Moreno-Reyes R, Kyrilli A, Lytrivi M, Bourmorck C, Chami R, Corvilain B. Is there still a role for thyroid scintigraphy in the workup of a thyroid nodule in the era of fine needle aspiration cytology and molecular testing? F1000Res. 2016; $5: 763$. 